\title{
On the Central Engine of short Gamma-ray Bursts
}

\author{
Stephan Rosswog ${ }^{1}$ and Enrico Ramirez-Ruiz ${ }^{2}$ \\ 1 Dep. Physics \& Astronomy, University of Leicester, Leicester LE1 7RH, UK \\ sro@astro.le.ac.uk \\ 2 Institute of Astronomy, Cambridge, CB3 OHA, UK enrico@ast.cam.ac.uk
}

\section{Introduction}

While there is so far no direct evidence linking coalescences of double neutron star systems to gamma-ray bursts (GRBs), there can be no doubt about the plausibility of this system, at least for the subclass of short GRBs. Neutron star binaries can provide huge reservoirs of gravitational binding energy and are expected to lead naturally to short overall durations with variations on millisecond time scales. In the following we want to assess two popular mechanisms to launch a GRB.

\section{Numerical method and simulations}

We have performed global, 3D simulations of the last stages prior to the coalescence and followed the subsequent hydrodynamical evolution for about 15 $\mathrm{ms}$. We use a temperature and composition dependent nuclear equation of state that covers the whole relevant parameter space in density, temperature and composition $[23,15]$. In addition, a detailed, multi-flavour neutrino treatment has been applied to account for energy losses and compositional changes due to neutrino processes. The neutrino treatment and the results concerning the neutrino emission have been described in detail in [17]. To solve the hydrodynamic equations we use the smoothed particle hydrodynamics method $(\mathrm{SPH})$, the simulations are performed with up to more than a million $\mathrm{SPH}$ particles, see Figure 1. The details of the production runs as well as those of several test runs can be found in $[15,17,18]$. Results focusing particularly on gamma-ray bursts have been presented in $[16,18,19]$.

\section{Assessment of the GRB mechanisms}

The energy released in a GRB represents just a minor fraction of the released gravitational binding energy (several times $10^{53} \mathrm{erg}$ ). This allows (in principle) 


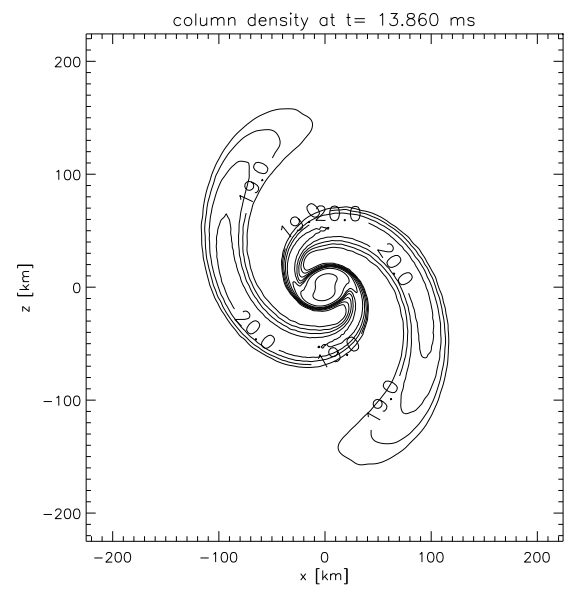

Fig. 1. Column density of an initially corotating neutron star binary system. For this simulation more than $10^{6} \mathrm{SPH}$ particles have been used.

for a plethora of possible burst mechanisms. The most popular of these are neutrino annihilation $[4,10,20,14,21,1,22]$ and the extraction of rotational energy via magnetic fields $[11,24,26,8,5,13,9]$.

\subsection{Neutrino Annihilation}

We find the merger remnant to emit neutrinos at a total luminosity of $\sim 2 \cdot 10^{53}$ erg/s. The luminosities are dominated by electron anti-neutrinos, followed by electron neutrinos and -slightly less luminous- the heavy lepton neutrinos. Their rms energies are around 15, 8 and $20 \mathrm{MeV}$, respectively. The bulk of the neutrino emission comes from the inner regions of the hot torus that has formed around the central object of the merger remnant (see [17] for details). In this context we consider two phenomena: (i) neutrinos and anti-neutrinos that annihilate above the merger remnant into $e^{ \pm}$pairs and (ii) like in the case of a newborn proto neutron star the neutrinos will blow off a strong baryonic wind from the remnant.

The thick disk with its geometry that is favourable for head-on neutrino collisions and its low baryon density along the original binary rotation axis launches a pair of relativistic jets [16]. An example of such a jet is shown in Figure 2. The typical energy provided in this way $\left(\sim 10^{48} \mathrm{erg}\right)$, however, is way below the isotropic energy estimates for short GRBs $\left(\sim 10^{51} \mathrm{erg}\right)$ at a redshift of $z=1[12,6]$ and therefore neutrino annihilation can only be a viable GRB mechanism if it goes along with a substantial collimation of the resulting outflow.

Such a collimation can be obtained via the ram pressure of the neutrinodriven, baryonic wind. This hydrodynamic collimation mechanism has been suggested by Levinson and Eichler [7]. They find that the jet half opening 


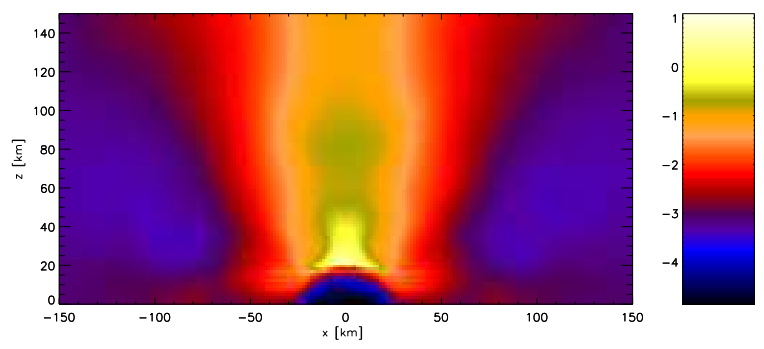

Fig. 2. Relativistic jet launched from a merger remnant via $\nu \bar{\nu}$ annihilation (upper half-plane; for details see [19]). Colour coded is the logarithm of the attainable Lorentz factor.

angle at large distances from the source, $\theta$, is determined by the ratio of jet and wind luminosity, $\theta \propto L_{j} / L_{w}$. Since the luminosity in the wind exceeds that of the jet by far, the outflow is collimated into a narrow solid angle. Using the theoretical neutron star mass distribution of [3], calculating the neutrino emission as a function of the binary system mass and parameterising the dependence of the wind luminosity as a function of the neutrino luminosity, $L_{w} \propto L_{\nu}^{\alpha}, \alpha=3.2 \ldots 3.4$ [25], we find the distributions of opening angles and luminosities shown in Figure 3. The broad distribution of opening angles is centred around 6 degrees, the luminosities around a few times $10^{50} \mathrm{ergs} / \mathrm{s}$. This is compatible with both the observational constraints on the luminosities of short GRBs and the estimated neutron star merger rates [18].

\subsection{Magnetic Processes}

The violent fluid motion within the merger remnant will almost certainly amplify the initial neutron star seed fields tremendously. To date, we are still lacking detailed MHD calculations of a neutron star coalescence, so we still have to wave our hands and draw conclusions from either simplified analytical models or purely hydrodynamic calculations.

The equipartition field strength, $B^{\mathrm{eq}}=\sqrt{8 \pi \rho c_{s}^{2}}$ in the central object of the merger remnant is (depending on the exact position) between $10^{16} \mathrm{G}$ and $10^{18} \mathrm{G}$, and $10^{14}$ to $10^{16} \mathrm{G}$ in the surrounding torus [19]. If just the relatively slow wrapping of the field lines via differential rotation (and no feedback onto the fluid) is assumed, equipartition will be reached in the central object within a few tens of seconds (provided it remains stable for long enough) and in the torus in around $4 \mathrm{~s}$. Other field amplification mechanisms 


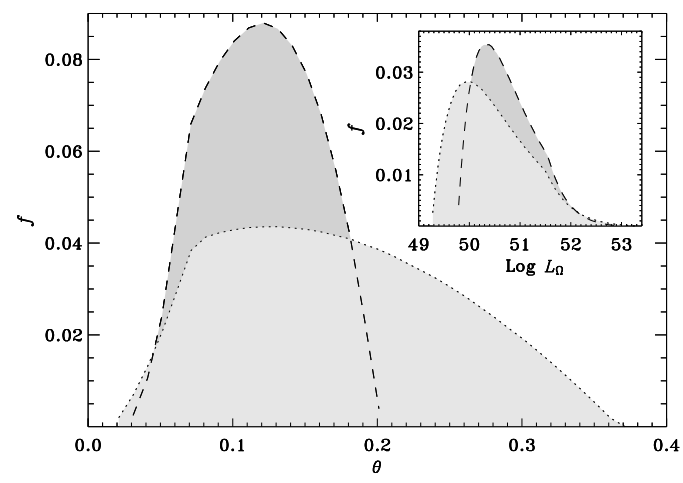

Fig. 3. Distribution of opening angles and apparent luminosities (inset) for GRBs launched via $\nu \bar{\nu}$ annihilation and collimated by means of a baryonic wind. (The dashed lines refer to $\alpha=3.2$, the dotted to $\alpha=3.4$; see text).

are expected to amplify the field exponentially leading to much shorter time scales. The fluid motion within the central object exhibits "convective cells" with sizes of $\sim 1 \mathrm{~km}$ and velocities of $\sim 10^{8} \mathrm{~cm} / \mathrm{s}$. Moreover, the neutrino emission will, like in a proto neutron star, establish a negative entropy and lepton number gradient and therefore drive vigorous convection (e.g. [2]). We find Rossby numbers (ratio of rotational and convective time scales), $R o \equiv T_{\text {rot }} / \tau_{\text {conv }}$, substantially below unity (down to 0.1 ) and therefore expect that the system can sustain a large scale dynamo. Such a dynamo will increase the magnetic field exponentially with an e-folding time close to the convective time scale. Using the numbers determined from our simulations we find that equipartition will be reached withing tens of milliseconds. The kinetic energy of the central object, $E_{\text {kin }}$, is large enough for an average field strength $\langle B\rangle_{\mathrm{co}}=\sqrt{3 \cdot E_{\mathrm{kin}} / R_{\mathrm{co}}^{3}} \approx 3 \cdot 10^{17} \mathrm{G}$, where $R_{\mathrm{co}}$ is the radius of the central object. With this average field strength the spin-down time scale is $\tau_{\mathrm{sd}}=E_{\mathrm{kin}} / L_{\mathrm{md}} \approx 0.2 \mathrm{~s}$, where $L_{\mathrm{md}}$ is the magnetic dipole luminosity, i.e. $\tau_{\mathrm{sd}}$ is of the order of the typical duration of a short GRB.

A discussion of further possible magnetic mechanisms can be found in [19]. All of these mechanisms yield luminosities in excess of a few times $10^{52} \mathrm{ergs} / \mathrm{s}$ and therefore yield typical time scales of order $1 \mathrm{~s}$.

\section{Conclusions}

We have assessed the two most popular mechanisms to produce short GRBs from the coalescence of neutron star binaries, namely the annihilation of neutrino anti-neutrino pairs and magnetic energy extraction mechanisms. We find 
that $\nu \bar{\nu}$ annihilation provides the driving stresses to launch a pair of relativistic, bipolar jets. To explain the expected isotropized energies the jets have to be narrowly collimated. This collimation can be provided by the energetic, neutrino-driven baryonic outflow that goes along with the coalescence. Due to the sensitivity to the neutrino luminosities even a narrow mass spectrum results in a broad distribution of opening angles and apparent GRB luminosities.

We further expect the initial neutron star magnetic fields to be amplified to values close to equipartition within fractions of a second. The estimates of the various investigated magnetic mechanisms all yield very large luminosities that do not require any beaming. If the current picture turns out to be correct, short GRBs should be composed of two distinct components: the one resulting from neutrino annihilation plus wind collimation is narrowly beamed and the other, much more energetic, and possibly uncollimated component results from the plethora of magnetic mechanisms.

\section{References}

1. Asano K., Fukuyama T., ApJ, 531, 949 (2000); Asano K., Fukuyama T., ApJ, 546, 1019 (2001)

2. R.I. Epstein, MNRAS, 188, 305 (1979)

3. Fryer C. L., Kalogera V., ApJ, 554, 548 (2001)

4. M. Jaroszinski, Acta Astronomica, 43, 183 (1993)

5. Katz, J.I., ApJ, 490, 772 (1997)

6. Lazzati D., Ramirez-Ruiz E., Ghisellini G., A\&A, 379, L39 (2000)

7. Levinson A., Eichler D., PhRvL, 85, 236 (2000)

8. Mészáros, P. \& Rees, M. J., ApJ, 482, L29 (1997)

9. Mészáros, P., Annu. Rev. Astron. Astrophys., 40, 137 (2002)

10. Mochkovitch R., Hernanz M., Isern J., Martin, X., Nature, 361, 236 (1993)

11. Narayan R., Paczyński B., Piran T., ApJ, 395, L83 (1992)

12. Panaitescu A., Kumar P., Narayan R., ApJ,561, L171 (2001)

13. Piran T., Physics Reports, 314, 575 (1999)

14. Popham R., Woosley S. E., Fryer C., ApJ, 518, 356 (1999)

15. S. Rosswog, M.B. Davies, MNRAS, 334, 481 (2002)

16. S. Rosswog, E. Ramirez-Ruiz, MNRAS, 336, L7 (2002)

17. S. Rosswog, M. Liebendörfer, MNRAS, 342, 673 (2003)

18. S. Rosswog, E. Ramirez-Ruiz, MNRAS, 343, L36 (2003)

19. S. Rosswog, E. Ramirez-Ruiz, M.B. Davies, MNRAS, in press (2003)

20. Ruffert M., Janka H.-T., Takahashi K., Schäfer G., A \& A, 319, 122 (1997)

21. Ruffert M., Janka H.-T., A \& A, 344, 573 (1999)

22. Salmonson J. D., Wilson, J. R., ApJ, 561, 950 (2001)

23. Shen H., Toki H., Oyamatsu K., Sumiyoshi K., Nuclear Physics, A 637, 435 (1998); Shen H., Toki H., Oyamatsu K., Sumiyoshi K., Prog. Theor. Phys., 100, 1013 (1998)

24. Thompson, C. \& Duncan, R.C., ApJ, 408, 194 (1993)

25. Thompson, T.A., Burrows,A., Meyer, B.S., ApJ, 562, 887 (2001)

26. Usov, V.V., Nature, 357, 472 (1992); Usov, V.V., MNRAS, 267, 1035 (1994) 